OTоLоGY

\title{
Acquisition of surgical skills for endoscopic ear and lateral skull base surgery: a staged training programme
}

\section{L'acquisizione degli skills chirurgici nella chirurgia endoscopica dell'orecchio medio e del basicranio laterale: un programma di apprendimento a stadi}

\author{
M. ALICANDRI-CIUFELLI ${ }^{2}$, D. MARCHIONI ${ }^{3}$, G. PAVESI ${ }^{2}$, F. CANZANO ${ }^{1}$, A. FELETTI ${ }^{2}$, L. PRESUTTI ${ }^{1}$ \\ ${ }^{1}$ Otolaryngology Head and Neck Surgery Department, University Hospital of Modena, Italy; ${ }^{2}$ Neurosurgery \\ Department, New Civil Hospital Sant'Agostino-Estense, Baggiovara, Modena, Italy; ${ }^{3}$ Otolaryngology Head and \\ Neck Surgery Department, University Hospital of Verona, Italy
}

\section{SUMMARY}

Endoscopic ear surgery is radically changing the treatment of several middle and inner ear pathology, but its learning presents well-known manual and technical difficulties. The aim of this paper is be to present a training programme based on a modular model of increased difficulties. The experience from 2007 to 2016 at the University Hospital of Modena and University Hospital of Verona was reviewed and analysed for further considerations and to establish stages of training. The increasing experience of expert surgeons who deal with this kind of surgery represented the main guide to establish the steps and progression of training. In addition, the potential risk of damage of vascular structures or nerves represents fundamental criteria for progression toward higher levels. Some not-mandatory skills were also suggested for stage of difficulty. Five stages of training were deemed appropriate for progressive learning of endoscopic ear and lateral skull base surgery, ranging from simple middle and external ear procedures to surgery of inner ear and internal auditory canal. Mastering of each level is suggested before attempting procedures at a higher level, in particular for procedures involving lateral skull base. Standardisation and adoption of modular incremental training are expected to facilitate improvement of otolaryngologists and neurosurgeons starting with endoscopic middle ear and lateral skull base surgery. Adherence to such a programme during the growth phase may potentially decrease the rate of complications, making the training programme safer.

KEY WORDS: Transcanal approach • Endoscopic ear surgery • Training program • Inner ear surgery $\bullet$ Lateral skull base

\section{RIASSUNTO}

La chirurgia endoscopica dell'orecchio sta cambiando radicalmente il trattamento di molte patologie dell'orecchio medio e interno ma il suo apprendimento presenta difficoltà manuali e tecniche. Lo scopo di questo lavoro è presentare un programma basato su un modello di apprendimento con difficoltà crescente. L'esperienza accumulata nell'Ospedale Universitario di Modena e in quello di Verona dal 2007 al 2016 è stata analizzata accuratamente con lo scopo di definire un programma di training chirurgico. I vari skills chirurgici sono stati definiti dai chirurghi esperti in questo campo. Il potenziale rischio di lesione delle strutture vascolari e nervose gioca un ruolo molto importante nella chirurgia otologica e per questo rappresenta un criterio fondamentale utile nella definizione dei diversi livelli di apprendimento chirurgico. Sono stati identificati 5 livelli di competenza in questo programma di apprendimento che, partendo dagli step chirurgici dell'orecchio esterno e dell'orecchio medio, arriva agli approcci chirurgici dell'orecchio interno e del basicranio. L'acquisizione di competenze progressivamente crescenti richiede necessariamente autonomia e padronanza degli skills chirurgici precedenti. Sono state riportate e discusse in una tabella riassuntiva tutte le informazioni relative ai pazienti operati durante il periodo analizzato. I risultati fanno riferimento al tasso di complicanze relativo agli interventi chirurgici di miringoplastica, timpanoplastica e di stapedoplastica in particolare al deficit del facciale, alle lesioni della dura madre e alla perdita uditiva. La curva di apprendimento degli otorinolaringoiatri e dei neurochirurghi che approcciano alla chirurgia endoscopica potrebbe essere standardizzata e ben definita da questo programma a stadi che, presupponendo l'acquisizione di competenze progressivamente crescenti, potrebbe essere utile anche a ridurre il tasso di complicanze.

PAROLE CHIAVE: Approccio transcanalare - Chirurgia endoscopica dell'orecchio $\bullet$ Programma di apprendimento $\bullet$ Chirurgia dell'orecchio interno $\bullet$ Basicranio laterale 


\section{Introduction}

Endoscopic instrumentation, techniques and knowledge have improved during the last few years, and we believe that, in the future, endoscopic surgical techniques will gain increasing importance in otologic surgery. From our 9-year experience in endoscopic ear surgery, we believe that most of the spaces considered to be of difficult accessibility with the microscopic technique can be easily visualised by endoscope-assisted surgery or by exclusive endoscopic approaches, and we feel that new anatomical and surgical concepts should be introduced for this.

A gradual introduction of endoscopic techniques to middle ear pathology treatment has taken place since the 1990 s $^{1}$. Endoscopy was used primarily for the visualisation of hidden areas such as the posterior epitympanum during classic microscopic tympanoplasties 2 . Gradually, it was also used in surgery to replace the microscope as the main tool during middle ear operations. Exclusive endoscopic tympanoplasty for cholesteatoma treatment was first described by Tarabichi et al. ${ }^{34}$. The new concept of endoscopic ear surgery redirected the attention away from the less critical areas (i.e. mastoid) toward the tympanic cavity and its "hard-to-reach" extensions. The endoscopic technique was codified for a minimally invasive eradication of limited attic cholesteatoma preserving the ossicular chain wherever possible, with complete removal of the disease. From this, a clinical application of the transcanal endoscopic approach has allowed to extend the indication of this technique to the cholesteatoma of the whole tympanic cavity without mastoid involvement.

At present, the main application of endoscopic surgery is in the surgical treatment of middle ear pathology, but with the natural evolution of the technique, there have also been advantages in lateral skull base diseases ${ }^{5}$. During the experience of recent years, present authors progressively noticed that the inner ear and the entire temporal bone can also be accessed in an endoscopic assisted fashion or even by exclusive endoscopic transcanal approaches. For the first time, an exclusive endoscopic approach to the internal auditory canal (IAC) was described ${ }^{6}$ and used to remove a cochlear schwannoma involving internal auditory canal (IAC) in March $2012^{6}$. The operation used a direct transcochlear approach from lateral to medial and from external to internal auditory canal, without any external incision.

Despite the several advantages compared to microscope, endoscopic ear surgery presents some technical and manual skills that are objectively difficult to acquire. This is due to several factors: the necessity of adequate hand-eye coordination; the very limited space of the surgical field; bleeding which prevents visualisation by obstructing the optic tip; some anatomical conditions (e.g. narrow external auditory canal (EAC)) which can limit the maneuvering of both optics and operative instruments.

In 2007 Snyderman et al. published an article that suggested a training program for acquisition of surgical skills in endonasal skull base surgery ${ }^{7}$. The authors standardised the training by suggesting a modular incremental training programme. Since endoscopic ear and lateral skull base surgery is a relatively new technique, a training program has not yet been established. The aim of this paper is to present a training programme based on modular model of increased difficulties, in a similar way of that already done for endoscopic skull base surgery.

\section{Materials and methods}

The experience from 2007 to 2016 (9 years) at the University Hospital of Modena and at the University Hospital of Verona was reviewed and a case series analysed for further considerations and to establish stages of training. The increasing experience of surgeons dealing with these types of surgeries represented the main guide to establish the steps and progression of training. In addition, the potential risk of damage to vascular structures and nerves represented a fundamental criteria for progression toward higher steps. The skills acquired by the authors before attempting that kind of surgery (e.g. microscopic ear surgery or endoscopic nasal surgery) were considered to establish their necessity before starting each level in endoscopic ear and lateral skull base surgery. Timeline and subjects of publications dealing with endoscopic ear surgery by our team were also reviewed and compared to confirm the progression throughout the stages. The stages were conceived and designed with the highest level of intrastage homogeneity of difficulty. Data from literature and data obtained from chart review of patients operated during the period analysed are also reported and discussed. In particular, the results were focused on complication rates to facial nerves, dura lesion and sensorineural hearing loss during myringoplasties, tympanoplasties and stapedoplasties.

\section{Results}

during the period of time selected, more than 500 endoscopic ear surgeries were performed at our institutions. Progression toward new procedures was made following a coronal plane from lateral to medial direction (Fig. 1). The beginning of the endoscopic ear surgery started with 


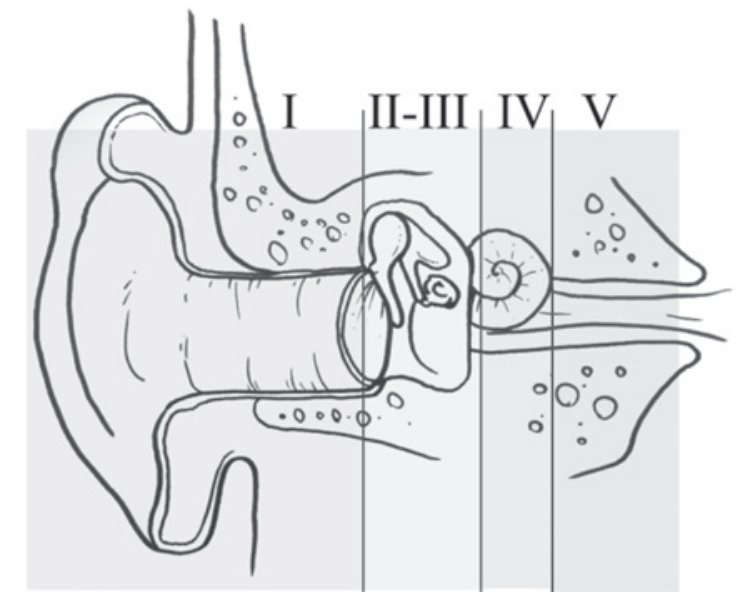

Fig. 1. Schematic drawing showing progression of training, in a coronal plane, from a lateral to a medial direction. Levels are indicated in roman numbers.

endoscopic-assisted procedures during microscopic tympanoplasties or myringoplasties ${ }^{289}$. Next, the first exclusive endoscopic procedures for cholesteatoma treatment were performed ${ }^{10}{ }^{11}$. The endoscopy was gradually implemented for other procedures such as tympanic facial nerve decompressions, and to improve former results such as in ossicular chain preservation ${ }^{12}$. With a sound experience acquired in middle ear surgery, and after a preliminary experience on cadavers, the first attempts to lateral skull base were then made, and the first cochlear schwannoma of the IAC was removed by an exclusive transcanal technique ${ }^{6}$. Then, more lateral skull base pathologies were treated by exclusive or combined techniques at our departments ${ }^{13}{ }^{14}$. Recently, the first case series of vestibular schwannomas of the IAC treated by an exclusive endoscopic technique (endoTTA) was published ${ }^{15}$. The timeline of publications, reflecting the increasing experience of our team is reported in Table I.

As mentioned in the methods, a staging system for training was designed based on two factors, which represented a fundamental criteria for progression toward higher steps: the increasing experience of surgeons which had been dealing with that kind of surgery, and the potential risk of damage of vascular structures or nerves.

Five stages were provided, as reported in Table II. In each stage operations that have a homogeneous grade of difficulty and risks are reported. Possible risks associated with each operation are reported in Table III. The complementary skills were also suggested by our team (Table IV).

Complication rates OVER the years obtained by chart review of patients operated between 2009 and 2016 were analysed. Our data documented a very low rate of unfavourable events for endoscopic middle ear surgery (Table V).

Results were focused on three different types of complications: facial nerve palsies, dura lesions and sensorineural hearing loss. Chart review of patients treated reported only three transitory facial nerve injuries, seven cases of postoperative sensorineural hearing loss and no dura lesions.

\section{Discussion}

Each type of surgery requires an adequate training period to familiarise the surgeon with anatomy and develop manual skills ${ }^{16}$. Skull base surgery is universally considered a very challenging district for neurosurgeons, maxillofacial and otolaryngologists, due to its complex anatomy and the very important structures that crosses it or are in close relationships with it (jugular vein, carotid artery, cranial nerves). In 2007, Snyderman et al. published an article that described the acquisition of surgical skills for endonasal skull base surgery, proposing a training programme ${ }^{7}$. The programme provided five stages of progression from "basic" procedures to very complex operations performed by exclusive endonasal approaches, starting from sphe-

Table I. Timeline of publication of new subjects dealing with endoscopic ear and lateral skull base surgery by our team.

\begin{tabular}{|c|c|}
\hline 2008 & Endoscopic-assisted tympanoplastie ${ }^{2}$ \\
\hline 2009 & Approaches to tensor fold area ${ }^{8}$, approaches to sinus tympani ${ }^{9}$ \\
\hline 2010 & Exclusive Endoscopic "closed" tympanoplasties ${ }^{10}$ \\
\hline 2011 & Exclusive Endoscopic "open" tympanoplasties ${ }^{11}$ \\
\hline 2012 & - \\
\hline 2013 & Pilot lateral skull base procedure ${ }^{13}$; first cochlear schwannoma treated by transpromontorial approach 6 ; benign neoplasms removal ${ }^{23}$ \\
\hline 2014 & Endoscopic cochlear implant procedure ${ }^{24}$; approach to geniculate ganglion ${ }^{21}$; approach to petrous apex lesions ${ }^{14}$ \\
\hline 2015 & Endoscopic cochlear implants in malformations ${ }^{25}$; pediatric tympanoplasties ${ }^{26}$ \\
\hline 2016 & $\begin{array}{l}\text { Case series of exclusive transcanal transpromontorial approach to internal auditory canal to treat vestibual schwannomas }{ }^{15} \text {; endoscopic } \\
\text { stapes surgery }{ }^{27}\end{array}$ \\
\hline
\end{tabular}


Table II. Transcanal endoscopic middle ear and lateral skull base training program.

\begin{tabular}{ll} 
Middle ear & Level I \\
& Office based endoscopies for diagnosis \\
& Myringotomies with/without \\
& tympanic tube placement \\
& Level II \\
& Combined approaches (endoscope assisted \\
& microscopic surgeries) \\
& Office base tympanic perforation repair \\
& Myringoplasties \\
& Endoscopic explorative tympanotomies \\
& with/without ossiculoplasties \\
& Level III \\
& Tympanoplasties for cholesteatoma \\
& Stapedoplasties \\
& Facial nerve decompression \\
& Endoscopic assisted cochlear implants \\
procedures & Endoscopic resection of middle ear neoplasms \\
(e.g. paragangliomas) & Level IV \\
\hline Inner ear & Endoscopic sovragenicular approaches \\
and lateral skull base & Infracochlear approaches \\
Transpromontorial approaches \\
for inner ear disease \\
Level V \\
EndoTTA for IAC pathology \\
\end{tabular}

Table III. Structures at risk.

Level I
No relevant risks
Level II
Ossicular chain
Level III
Ossicular chain, facial nerve, labyrinth
Level IV
Facial nerve, labyrinth, Jugular bulb, carotid artery
Level V
Facial nerve, jugular bulb, carotid artery, lower cranial nerves, vessels of
CPA (AICA), brainstem. Risk of post-operative CSF leak

noethmoidectomies (level I) to reach the highest level of difficulty (level V) which included treatment of aneurisms and highly vascularised tumours. The first levels (I and II) are those that are expected to be achieved within residency, then the program progresses in complexity.
Table IV. Complimentary expertise.

Level I
Endoscopic nasal surgery
Level II
Microscopic middle ear surgery
Level III
Microscopic middle ear surgery
Level IV
Microscopic transpetrous approaches
Level V
Acoustic neuroma surgery (retrosigmoid, translabyrinthine approaches)

Based on the substantial expertise of the authors, a staged training programme for acquisition of surgical skills for endoscopic middle ear and lateral skull base surgery is herein presented, strongly adhering to the same methods and principles used by Snyderman et al. As mentioned in the Results, the training programme described provides progression in a coronal plane, and with a lateral to medial direction, reflecting the complexity and presence of vital structures of the lateral skull base (Fig. 1).

Publications considered in Table I only deal specifically with surgery, and only those in which for the first time a new experience was described. Publications dealing exclusively with anatomy, physiologic issues, or reviews were excluded from this list. It can be noticed that they substantially reflected the progression proposed in Table II. The overall number of publications from 2007 to 2016 on the topic of endoscopic middle ear and lateral skull base by our team surpassed 40 , and this is to testify the increasing interest by scientific community on that topic.

As reported in Table II, level I of the training programme presented includes office based explorations of EAC and tympanic membrane or tympanic tube placement. In fact, those basic procedures allow the surgeon to obtain the first impressions about depth of field, hand-eye coordination, magnification and surgical manoeuvring using the endoscope. The risks of severe complications in this kind of procedures are virtually absent. Prior expertise in endoscopic nasal surgery can be very helpful at this stage, although not mandatory, to acquire skills.

This step can be attempted very early, from the beginning of residency, during normal office evaluations or followup in the outpatient clinic. In addition, endoscopic ear dewaxing can be a good exercise at this level. When enough confidence is obtained, level II can be approached. In level II, the surgeon can start using the endoscope for more operative procedures, also attempting its use inside the 
Table V. Complication rates in patients operated by EES between 2009 and 2016.

\begin{tabular}{lcccccc} 
& 2011 & $\mathbf{2 0 1 2}$ & $\mathbf{2 0 1 3}$ & $\mathbf{2 0 1 4}$ & $\mathbf{2 0 1 5}$ & $\mathbf{2 0}$ \\
Dura lesion & 0 & 0 & 0 & 0 & 0 & 1 \\
SNHL * $^{\text {Facial palsy }}$ & 1 & 0 & 1 & 3 & 0 & 1 \\
\hline
\end{tabular}

${ }^{*}=$ hearing loss higher than $30 \mathrm{~dB}$ in at least one frequency.

tympanic cleft, for example in combined microscopic/endoscopic approaches ${ }^{2}$. This can further improve manual skills, and also provide knowledge of instruments to operate inside the middle ear. Moreover, it allows surgeon to become familiarsed with angled optics, which can be used to explore sinus tympani, tensor fold area, protympanic spaces, looking for cholesteatoma residuals ${ }^{89}$ during microscopic surgeries. Level II can also include myringoplasties or explorative tympanotomies, made by exclusive endoscopic approaches. In these operations, there is a potential risk of damage to the ossicular chain, and for this some experience must have been acquired at level I. In level II bleeding management is very important: in fact, due to the EAC skin incision and flap elevation, the surgeon will acquire the ability in bleeding control, and avoid dirtying the endoscopic tip. This is probably the most difficult skill to acquire in endoscopic ear and lateral skull base surgery, and in most cases it can discourage the surgeon in training. Bleeding control requires patience and good manual skills, but also knowledge in the positioning of the patient, cooperation with the anaesthesiologist in keeping blood pressure low and an appropriate technique in raising the tympanomeatal flap, which could include also the use of hot blades. These factors are considered fundamental for progressing toward the next levels.

Level III provides exclusive approaches to treat typical pathologies of the middle ear (e.g. cholesteatoma), and more rare pathologies that could have risks of damage to the ossicular chain, facial nerve or labyrinth. Although it is very likely that adequate expertise is obtained by the surgeon in training after levels I and II, expertise in microscopic procedures is anyway advisable, because in case of problems during surgery or in particularly difficult procedures, a possible shift to the microscopic vision and bimanuality can be very helpful. Level III represents the final step for middle ear endoscopic surgery but similar considerations can be done with level IV, where lateral skull base procedures are attempted (Figs. 2, 3).

As already documented in former articles ${ }^{5}$, endoscopic approaches to lateral skull base are less invasive compared to classic microscopic procedures. Nonetheless, at this level the former experience in microscopic approaches can be very helpful in case of necessity, due to the fact that even more delicate structures will be manipulated (fa-

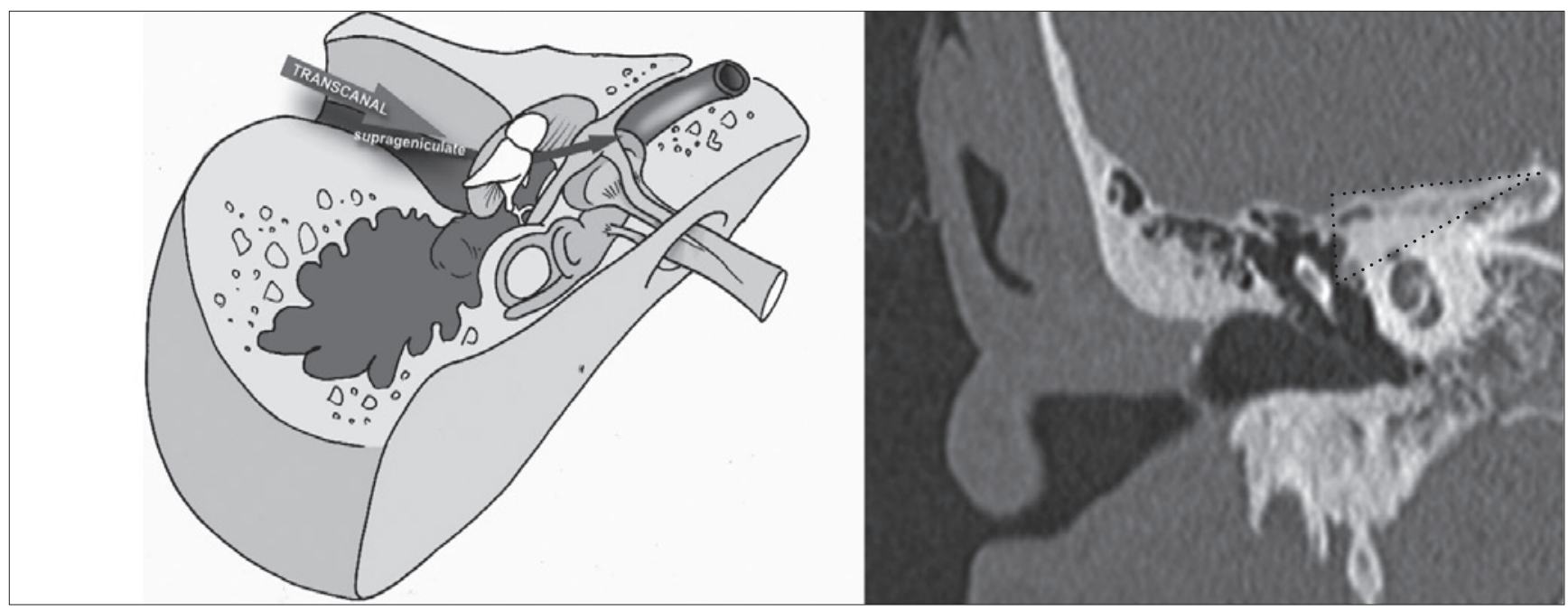

Fig. 2. The transcanal endoscopic suprageniculate approach: on the left the schematic drawing shows the surgical route from the external auditory canal to the suprageniculate fossa (black arrow); on the right, the CT scan in coronal view shows the working area and bony removal (dotted area) which may be performed under this approach between the middle cranial fossa and the facial nerve into the petrous apex over the cochlea (from Marchioni et al., $2013{ }^{5}$, mod.). 


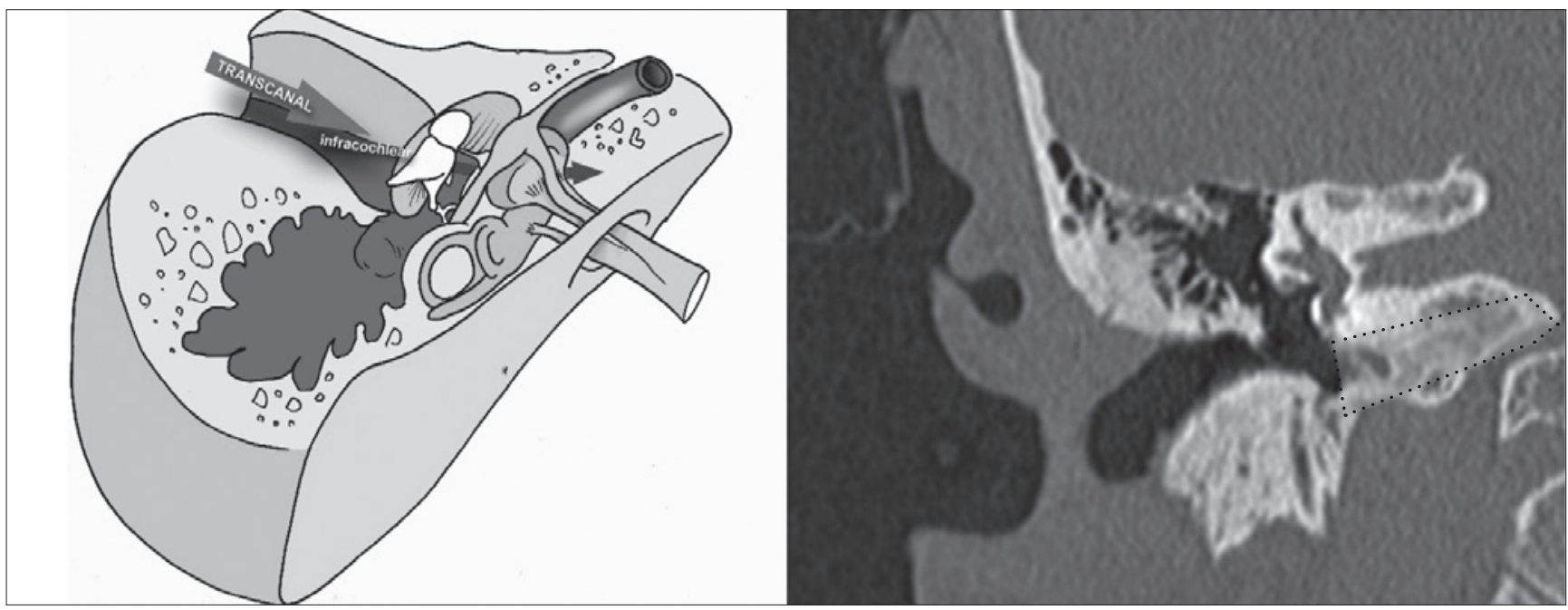

Fig. 3. The transcanal endoscopic infracochlear approach: on the left, the schematic drawing shows the surgical route from the external auditory canal to the infracochlear below the cochlea (black arrow); on the right, the CT Scan in coronal view shows the working area, and the bony removal (dotted area), which may be performed under this approach between the cochlea superiorly and the jugular bulb inferiorly in order to reach the petrous apex cells (from Marchioni et al., $2013^{5}$, mod.).
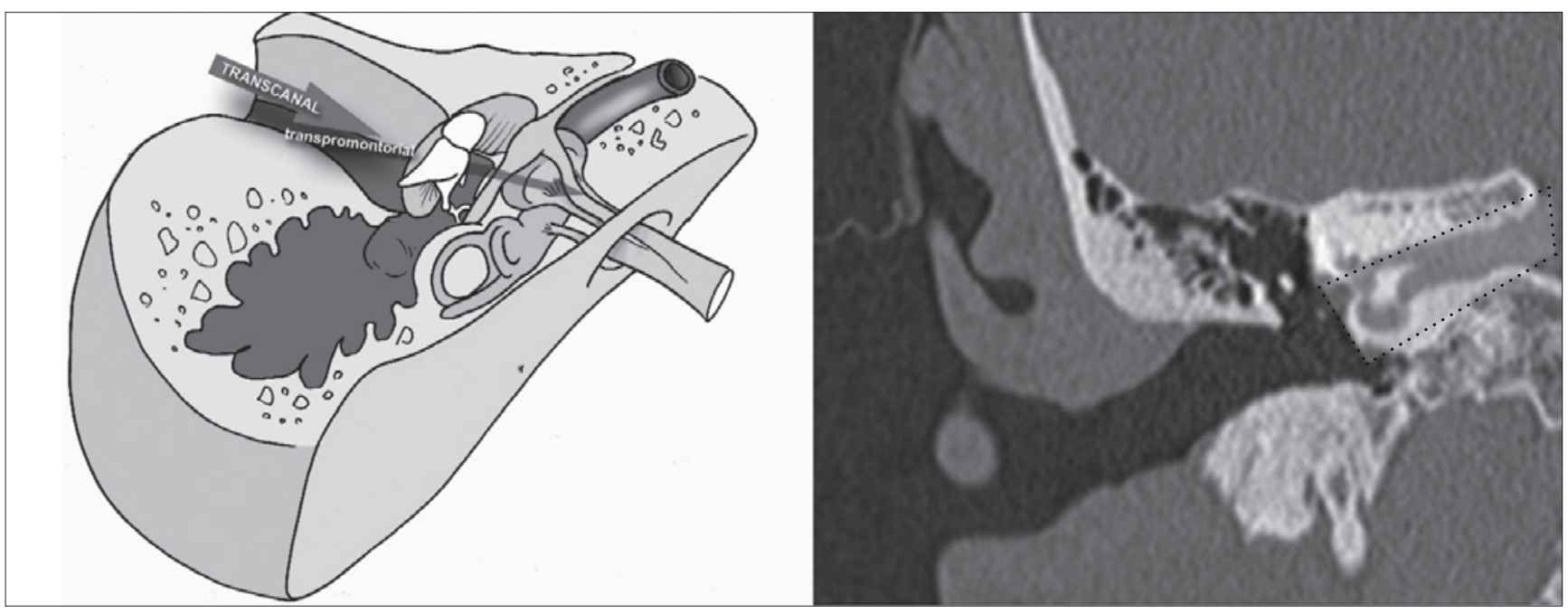

Fig. 4. The transcanal endoscopic transpromontorial approach: on the left, the schematic drawing shows the surgical route from the external auditory canal to the fundus of the IAC through the promontory (black arrow); on the right the CT Scan in coronal view shows the working area, and the bony removal (dotted area) which may be performed under this approach through the promontory, removing the cochlea and the vestibule, reaching the fundus of the IAC (from Marchioni et al., $2013^{5}$, mod.).

cial nerve, labyrinth, jugular bulb, carotid artery), and due to the risks of a possible damage.

In level V (Fig. 4), where the surgeon reaches the IAC and CPA, he/she must be aware not only of the complex anatomy of CPA with all the posterior fossa vascularisation and cranial nerves. Actually, it is also advisable to have former experience with CSF leak management and reconstruction procedures of lateral skull base to avoid post-operative complications related to it. Moreover, strong expertise in dissecting the vestibular schwannoma can be helpful due to the fact that in exclusive endoscopic approaches it should be done by only one hand, as described in the endoscopic transcanal traspromontorial approach to IAC ${ }^{15}$.

Although the acquired skills suggested (Table IV) before attempting every level can be nowadays important, the authors do not consider them as mandatory. This is due to the fact that the evolution of middle ear and lateral skull 
base is at present very difficult to predict, and will mostly depend on development of technologies ${ }^{17}$, instrumentation ${ }^{18-20}$ and consequent evolution and modification of the technique. It is not unlikely that future generations will begin their expertise in this surgical field using directly and exclusively the endoscope, so for example the expertise in microsurgery could progressively become less necessary. Anyway, at present, the acquired skills mentioned will certainly increase the proficiency and safety in performing some operations, and may also very likely shorten the progression time.

Another very important point, as already underlined by Snyderman et al. ${ }^{7}$, is that although learning occurs mostly in the operating theatre, it is necessary that a sufficient amount of time be spent in the dissection laboratory. This should include both basic and advanced dissection courses, possibly focused on endoscopic middle ear and lateral skull base surgery, which are starting nowadays to be organised worldwide.

Actually, the long learning curve required to master endoscopic ear surgery urges the search for models to practice it. Many studies have demonstrated the validation of an animal model as a proper training model for this technique. In the experimental study reported by Anschuetz et al., the authors assessed the suitability of an ovine model for training in exclusive endoscopic surgery, assessing the time need for dissection and procedures. A significant improvement in all the surgical steps was demonstrated comparing mean surgical time required to perform them ${ }^{28}$.

Simulators can also provide a challenging, safe and controlled environment to master the basic skills needed to perform endoscopic ear surgery. Several simulators for training of endoscopic techniques and procedures have been developed. Surgeons receiving simulator training show significant differences between first and last completion time with the dominant hand and average completion time for junior and senior residents suggesting construct validity. A 3-dimensional (3D) printed endoscopic ear surgery simulator may facilitate the development of surgical skills with high fidelity and low cost ${ }^{29}$.

The simulator is considered useful for training eye-hand coordination for endoscopic surgery. However, the use of simulators in training hospitals is limited. This may be partly due to their high cost andextensive system requirements.

Neuronavigation systems can be applied in lateral skull base surgery to facilitate surgeons during training. Neuronavigation is very useful to obtain information during surgical procedures such as vascular details, cranial nerve positions and exact size and position of ENT tumours. Endoscopic approaches to the lateral skull base are the last step during endoscopic ear surgery training program, so in particular for trainees, neuronavigation could help in situations with altered anatomy, such as revision cases, or in case of far advanced pathology that could alter completely anatomy.

Actually, as detailed in the results, complication rates in middle ear endoscopic surgery is very low. The surgery analysed was only that regarding middle ear procedures so as to obtained a significant number of patients. Although procedures included were relevant, complication rates were so low that eventually it was not possible to draw any meaningful timeline that could show improvement or worsening of surgeon performances.

As mentioned before, EES is a relatively new technique but results have been progressively reported in literature. Improvement of the technique lead to results that can be compared, at least in term of pathology eradication, to microscopic techniques. This was also reported by Presutti et al. whose literature review focused on outcomes obtained using endoscopic surgery for treatment of middle ear cholesteatoma. The authors analysed 515 patients treated exclusively with endoscope or with a combined procedure: the rates of recurrence and residual disease $(9.3 \%)$ were similar than those obtained with microscopic approaches ${ }^{30}$.

Even in the experience of our institution favourable results were obtained for endoscopic treatment of cholesteatoma at a mean follow-up of 5 years. In total 234 patients were treated: 144 underwent an exclusive endoscopic approach, whereas 100 were treated with a combined approach with mastoidectomy. Based on that analyses, $68 \%$ of patients treated were free from disease during postoperative follow-up visits; $12 \%$ were diagnosed a recurrence and $20 \%$ had residual disease ${ }^{31}$.

The experiences reported in authors' opinion demonstrate that training in EES is not associated with significant worsening of prognosis or complication rates, at least if a staged training programme is followed. In our institution, roughly 9 years were required to reach the IAC starting from basic procedures, which we would consider an adequate amount of time. Faster training could be potentially less safe, in particularly for mastering lateral skull base approaches.

In the authors' opinion, cooperation of otolaryngologists and neurosurgeons during the whole period of learning is strongly advisable. Otolaryngologists would particularly benefit from cooperation with neurosurgeons when attempting endoscopic lateral skull base procedures (levels IV and V). On the contrary, in the first level (from I to III) the neurosurgeon would strongly benefit from the otolaryngologist in understanding middle ear anatomy, landmarks and knowledge of middle ear surgery instru- 
mentation. Standardisation and adoption of a modular incremental training are expected to facilitate the improvement of both otolaryngologists and neurosurgeons starting with endoscopic middle ear and lateral skull base surgery.

\section{Conclusions}

Five stages of training are deemed appropriate for progressive learning of endoscopic ear and lateral skull base surgery, from simple middle ear procedures to surgery of inner ear and IAC. Mastering of each level is suggested before attempting procedures at a higher level, in particular for procedures involving the inner ear and lateral skull base. Standardisation and adoption of a modular incremental training are expected to facilitate improvement of otolaryngologists and neurosurgeons starting these types of surgeries.

\section{References}

1 Thomassin JM, Korchia D, Doris JM. Endoscopic guided otosurgery in the prevention of residual cholesteatomas. Laryngoscope 1993;103:939-43.

2 Presutti L, Marchioni D, Mattioli F, et al. Endoscopic management of acquired cholesteatoma: our experience. J Otolaryngol Head Neck Surg 2008;37:481-7.

3 Tarabichi M, Nogueira JF, Marchioni D, et al. Transcanal endoscopic management of cholesteatoma. Otolaryngol Clin North Am 2013;46:107-30.

4 Marchioni D, Alicandri-Ciufelli M, Rubini A, et al. Endoscopic transcanal corridors to lateral skull base: first experiences. Laryngoscope 2015;125:1-13.

5 Presutti L, Alicandri-Ciufelli M, Cigarini E, et al. Choclear schwannoma removed trough the external auditory canal by a transcanal exclusive endoscopic technique. Laryngoscope 2013;123:2862-7.

6 Snyderman C, Kassam A, Carrau R, et al. Acquisition of surgical skills for endonasal skull base surgery: a training program. Laryngoscope 2007;117:699-705.

7 Marchioni D, Mattioli F, Alicandri-Ciufelli M, et al. Endoscopic approach to tensor fold in patients with limited attic cholesteatoma. Acta Otolaryngol 2008;25:1-9.

8 Marchioni D, Mattioli F, Alicandri-Ciufelli M, et al. Transcanal endoscopic approach to the sinus timpani: a clinical report. Otol Neurotol 2009;30:758-65.

9 Marchioni D, Alicandri-Ciufelli M, Molteni G, et al. Endoscopic tympanoplasty in patients with attic retraction pockets. Laryngoscope 2010;120:1847-55.

10 Marchioni D, Villari D, Alicandri-Ciufelli M, et al. Endoscopic open technique in patients with middle ear cholesteatoma. Eur Arch Otorhinolaringol 2011;268:1557-63.

11 Marchioni D, Alicandri-Ciufelli M, Molteni G, et al. Ossicular chain preservation after exclusive endoscopic transcanal tympanoplasty: preliminary experience. Otol Neurotol 2011;3:626-31.

12 Presutti L, Nogueira JF, Alicandri-Ciufelli M, et al. Beyond the middle ear: endoscopic surgical anatomy and approaches to inner ear and lateral skull base. Otolaryngol Clin North Am 2013;46:189-200.

13 Presutti L, Alicandri-Ciufelli M, Gioacchini FM, et al. Combined lateral microscopic/endoscopic approaches to petrous apex lesions: pilot clinical experiences. Ann Otol Rhinol Laryngol 2014;123:550-9.

14 Marchioni D, Alicandri-Ciufelli M, Rubini A, et al. Exclusive endoscopic transcanal transpromontorial approach: a new perspective for internal auditory canal vestibular schwannoma treatment. J Neurosurg 2017;126:98-105.

15 Pothier DD. Introducing endoscopic ear surgery into practice. Otolaryngol Clin North Am 2013;46:245-55.

16 Kakehata S, Watanabe T, Ito T, et al. Extension of indications for transcanal endoscopic ear surgery using an ultrasonic bone curette for cholesteatomas. Otol Neurotol 2014;35:101-7.

17 Khan MM, Parab SR. Endoscopic cartilage tympanoplasty: a two-handed technique using an endoscope holder. Laryngoscope 2016;126:1893-8.

18 Badr-El-Dine M, James AL, Panetti G, et al. Instrumentation and technologies in endoscopic ear surgery. Otolaryngol Clin North Am 2013;46:211-25.

19 Chan JY, Leung I, Navarro-Alarcon D, et al. Foot-controlled robotic-enabled endoscope holder for endoscopic sinus surgery: a cadaveric feasibility study. Laryngoscope 2016;126:566-9.

20 Marchioni D, Alicandri-Ciufelli M, Piccinini A, et al. Surgical anatomy of transcanal endoscopic approach to the tympanic facial nerve. Laryngoscope 2011;121:1565-73.

21 Marchioni D, Alicandri-Ciufelli M, Nogueira JF, et al. From external to internal auditory canal: surgical anatomy by an exclusive endoscopic approach. Eur Arch Otorhinolaryngol 2013;270:1267-75.

22 Marchioni D, Alicandri-Ciufelli M, Gioacchini FM, et al. Transcanal endosocpic treatment of middle ear benign neoplasms. Eur Arch Otorhinolaryngol 2013;270:2997-3004.

23 Marchioni D, Grammatica A, Alicandri-Ciufelli M, et al. Endoscopic cochlear implant procedure. Eur Arch Otorhinolaryngol 2014;271:959-66.

24 Marchioni D, Soloperto D, Guarnaccia MC, et al. Endoscopic assisted cochlear implants in ear malformations. Eur Arch Otorhinolaryngol 2015;272:2643-52.

25 Marchioni D, Soloperto D, Rubini A, et al. Endoscopic exclusive transcanal approach to the tympanic cavity cholesteatoma in pediatric patients: our experience. Int J Pediatr Otorhinolaryngol 2015;79:316-22.

26 Marchioni D, Soloperto D, Villari D, et al. Stapes malformations: the contribute of the endoscopy for diagnosis and surgery. Eur Arch Otorhinolaryngol 2016;273:1723-9.

27 Anschuetz L, Bonali M, Ghirelli M, et al. An ovine model for 
exclusive endoscopic ear surgery. J Otolaryngol Head Neck Surg 2017; 143:247-52.

28 Barber SR, Kozin ED, Dedmon M, et al. 3D-printed pediatric endoscopic ear surgery simulator for surgical training. Int J Pediatr Otorhinolaryngol 2016;90:113-8.

29 Presutti L, Gioacchini FM, Alicandri-Ciufelli M, et al. $R e$ - sults of endoscopic middle ear surgery for cholesteatoma treatment: a systematic review. ACTA Otorhinolaryngol Ital 2014;34:153-7.

30 Alicandri-Ciufelli M, Marchioni D, Kakehata S, et al. Endoscopic management of attic cholesteatoma, long term results. Otolaryngol Clin N Am 2016;49:1265-70.

Received: July 17, 2017 - Accepted: October 15, 2017

Address for correspondence: Federica Canzano, Otolaryngology Head and Neck Surgery Department, University Hospital of Modena, via del Pozzo 71, 41100 Modena, Italy. Tel. +39 0594222402. Fax +390594 222454. E-mail: federica.canzano@gmail.com 